\title{
A INFLUÊNCIA DOS ÁCIDOS HEXENURÔNICOS NO RENDIMENTO E NA BRANQUEABILIDADE DA POLPA KRAFT
}

Gustavo Ventorim* e José Cláudio Caraschi

Departamento de Engenharia Industrial Madeireira, Universidade Estadual Paulista "Júlio de Mesquita Filho", Rua Geraldo Alckmin, 519, 18409-010 Itapeva - SP, Brasil

Jorge Luiz Colodette e José Lívio Gomide

Departamento de Engenharia Florestal, Universidade Federal de Viçosa, 36571-000 Viçosa - MG, Brasil

Recebido em 26/3/08; aceito em 8/10/08; publicado na web em 2/2/09

\begin{abstract}
THE INFLUENCE OF HEXENURONIC ACIDS IN YIELD AND BLEACHABILITY OF PULP KRAFT. This study investigated the impact of pulp hexenuronic acids (HexAs) content on pulping yield by changing cooking reaction temperature. The bleachability of pulps containing variable amounts of HexAs was also investigated. The cooking at $170^{\circ} \mathrm{C}$ produced pulp of kappa number, HexAs and screen yield of $16.2,49.4 \mathrm{mmol} / \mathrm{kg}$ and $50.2 \%$, respectively, whereas the cooking at $156{ }^{\circ} \mathrm{C}$ resulted pulp of kappa $17.0,61.3$ $\mathrm{mmol} / \mathrm{kg}$ HexAs and 50.8\% screened yield. The pulp produced at lower cooking temperature also showed better bleachability as evaluated by the total amount of active chlorine required to achieve $90 \%$ ISO. The sequence $\mathrm{OA}_{\mathrm{HT}} \mathrm{D}(\mathrm{EP}) \mathrm{DD}$ showed the lowest bleaching performance among all.
\end{abstract}

Keywords: hexenuronic acid; pulping kraft; ECF bleaching.

\section{INTRODUÇÃO}

As principais estruturas de ácido carboxílico que ocorrem nos polissacarídeos da madeira são os grupos de ácidos glicurônicos, usualmente na forma dos ácidos mono metil éter, 2-O-(4-metil- $\alpha$-Dglicopiranosilurônico) e galacturônico.

O conteúdo total de unidades de ácidos urônicos presentes na madeira está em torno de 4-5\% da madeira seca. ${ }^{1}$ Enquanto as unidades de ácido glicurônico predominam nas xilanas (hemiceluloses) presentes na madeira, as de ácido galacturônico são constituintes das pectinas. Como as substâncias pécticas estão localizadas, principalmente, na lamela média e na parede primária, são facilmente dissolvidas durante a deslignificação da madeira. ${ }^{1}$ Os ácidos hexenurônicos são formados durante a polpação alcalina pela modificação dos ácidos 4-O-metilglicurônicos, presentes nas xilanas. ${ }^{2,3}$ A Figura 1 mostra o mecanismo de formação dos ácidos hexenurônicos.

As condições de polpação que mais influenciam o conteúdo de ácidos hexenurônicos na polpa são álcali ativo, sulfidez e temperatura. ${ }^{4,5}$ A Figura 2 mostra o mecanismo da degradação dos ácidos hexenurônicos em meio alcalino. A concentração de ácido hexenurônico em polpas de diversas madeiras de folhosas em diferentes condições de cozimento kraft e, também, a sua concentração na polpa depende de vários fatores, como tempo de cozimento, temperatura

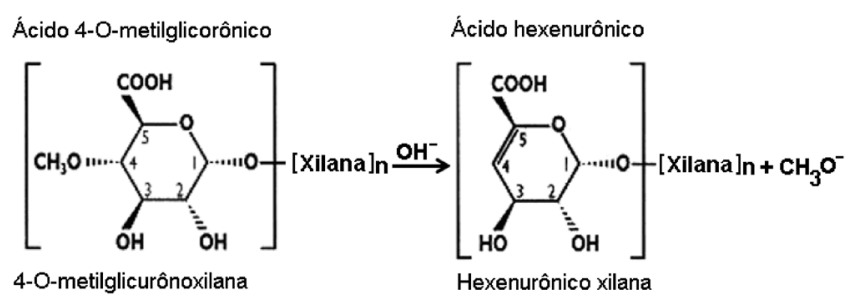

Figura 1. Conversão do 4-O-metil-glicuronoxilana em hexenuronoxilana durante a polpação kraft

*e-mail: ventorim@itapeva.unesp.br

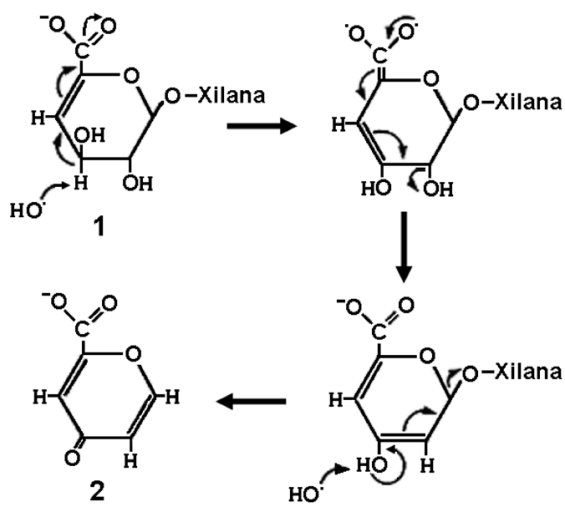

Figura 2. Mecanismo da degradação dos ácidos hexenurônicos sob condições de polpação alcalina

e carga de álcali. ${ }^{6}$ Os mesmos autores verificaram, ainda, que dois mecanismos afetam o decréscimo do teor de ácidos hexenurônicos, a hidrólise alcalina e conseqüente separação dos grupamentos de ácidos hexenurônicos das cadeias de xilana e a dissolução das xilanas, provocando decréscimo no rendimento. ${ }^{5,6}$ Há indicações de que podem proteger as xilanas contra as reações de despolimerização terminal; portanto, sua presença na polpa preserva o rendimento em etapas alcalinas.

Os ácidos hexenurônicos não são desejáveis nos processos de branqueamento pois, além de consumirem os reagentes químicos do branqueamento, causam um aumento na sua habilidade de quelar metais, comparado com seu precursor, o ácido 4-O-metil glicurônico e, conseqüentemente, uma maior reversão de alvura. ${ }^{\text {? }}$

Os ácidos hexenurônicos não são reativos no branqueamento alcalino com oxigênio e peróxido de hidrogênio. Tem sido demonstrado que a fração de xilanas dissolvidas (extraíveis em sulfóxido de dimetila) durante o branqueamento com peróxido de hidrogênio contém quantidades significativas de ácidos hexenurônicos (4-6 mol por 100 mol de xilose), o que confirma que estes ácidos são estáveis em relação ao peróxido. ${ }^{8}$ 
Apenas os reagentes de branqueamento que promovem o ataque eletrofílico $\left(\mathrm{O}_{3}, \mathrm{Cl}_{2}\right.$ e outros) são capazes de remover os ácidos hexenurônicos; mas, o estágio de branqueamento com dióxido de cloro também remove os ácidos hexenurônicos, devido aos reagentes formados durante o estágio de branqueamento.

Uma outra maneira de remover os ácidos hexenurônicos é utilizando um estágio de hidrólise ácida. Em geral, ambos os grupos (enol-éter e ácido carboxílico insaturado) são inertes sob condições ligeiramente ácidas. Sob condições ácidas fortes, os grupos de enoléter sofrem hidrólise rápida, levando à formação de aldeído ou cetona e álcool. ${ }^{9}$ No entanto, a hidrólise em meio ácido, mesmo intensificada $\left(1 \mathrm{~h}, 110^{\circ} \mathrm{C}\right.$ e pH 3,5), não resulta em completa degradação dos ácidos hexenurônicos em polpas kraft. ${ }^{4}$

O objetivo deste trabalho foi avaliar a influência do teor de ácidos hexenurônicos na polpa celulósica produzida pela polpação kraft em diferentes temperaturas $\left(170\right.$ e $\left.156^{\circ} \mathrm{C}\right)$, em função do rendimento do processo e da braqueabilidade dessas polpas, utilizando as sequiências de branqueamento $\mathrm{OD}(\mathrm{E}+\mathrm{P}) \mathrm{DD}, \mathrm{OA}_{\mathrm{HT}} \mathrm{D}(\mathrm{E}+\mathrm{P}) \mathrm{DD}, \mathrm{OD}(\mathrm{E}+\mathrm{P}) \mathrm{DP}$ e $\mathrm{OA}_{\mathrm{HT}} \mathrm{D}(\mathrm{E}+\mathrm{P}) \mathrm{DP}$.

\section{PARTE EXPERIMENTAL}

\section{Material}

Foi utilizada uma amostra representativa da madeira de Eucalyptus grandis com 7 anos de idade, proveniente da região de Itapeva-SP. Sendo dividido em duas fases: polpação química e branqueamento ECF.

\section{Métodos}

\section{Polpação química pelo processo kraft}

Foram realizados diversos cozimentos convencionais utilizando as seguintes condições: massa de cavacos - $250 \mathrm{~g}$; temperatura - 170 ${ }^{\circ} \mathrm{C}$; tempo até a temperatura - $90 \mathrm{~min}$; tempo na temperatura - 60 min; sulfidez - $25 \%$ e relação licor:madeira: $4: 1$. Foi realizada uma curva de álcali ativo $\left(12,14,16\right.$ e $18 \%$ como $\left.\mathrm{Na}_{2} \mathrm{O}\right)$ para atingir um número kappa de $17 \pm 1$. Em seguida, foi utilizado um álcali ativo de $17 \%$ para produzir as polpas de número kappa 17.

Foi realizado um cozimento convencional com o mesmo fator $\mathrm{H}$ utilizando as seguintes condições: temperatura $-156^{\circ} \mathrm{C}$; tempo até a temperatura - $90 \mathrm{~min}$; tempo na temperatura - $214 \mathrm{~min}$; álcali ativo $17 \%$ como $\mathrm{Na}_{2} \mathrm{O}$; sulfidez - $25 \%$ e relação licor:madeira: 4:1.

Os cozimentos foram realizados em uma autoclave, marca Regmed, com quatro reatores individuais de capacidade de $2 \mathrm{~L}$ cada, com perfil de temperatura controlado por computador.

\section{Branqueamento}

Foram realizadas quatro diferentes sequiências de branqueamento pelo processo elementary chlorine free $(\mathrm{ECF})$ : $\mathrm{OD}(\mathrm{E}+\mathrm{P}) \mathrm{DD}$, $\mathrm{OA}_{\mathrm{HT}} \mathrm{D}(\mathrm{E}+\mathrm{P}) \mathrm{DD}, \mathrm{OD}(\mathrm{E}+\mathrm{P}) \mathrm{DP}$ e $\mathrm{OA}_{\mathrm{HT}} \mathrm{D}(\mathrm{E}+\mathrm{P}) \mathrm{DP}$, cujas condições estão apresentadas na Tabela 1.

Tabela 1. Condições gerais de branqueamento

\begin{tabular}{lccccccc}
\hline \multirow{2}{*}{ Condição } & \multicolumn{7}{c}{ Estágios de branqueamento } \\
& $\mathrm{O}$ & $\mathrm{A}_{\mathrm{HT}}$ & $\mathrm{D}$ & $(\mathrm{E}+\mathrm{P})$ & $\mathrm{D}$ & $\mathrm{D}$ & $\mathrm{P}$ \\
\hline Consistência (\%) & 10 & 10 & 10 & 10 & 10 & 10 & 10 \\
Tempo (min) & 60 & 120 & 30 & 60 & 180 & 180 & 120 \\
Temperatura $\left({ }^{\circ} \mathrm{C}\right)$ & 95 & 95 & 60 & 75 & 70 & 70 & 90 \\
$\mathrm{pH}$ & 12,0 & 3,5 & 3,0 & 11,0 & 3,8 & 4,5 & 10,5 \\
Pressão $(\mathrm{kPa})$ & 500 & - & - & - & - & - & - \\
\hline
\end{tabular}

\section{Procedimentos analíticos}

As normas dos procedimentos realizados neste estudo foram: Número Kappa (TAPPI T $236 \mathrm{~cm}-85) ;{ }^{10}$ formação de folha para medição de alvura (CPPA C.5); ${ }^{11}$ alvura (TAPPI T 452 om-86); viscosidade (TAPPI T 230 om-89); análises dos teores dos ácidos hexenurônicos na polpa marrom e da polpa após o estágio de hidrólise ácida a quente $;^{4}$ determinação da concentração de reagentes utilizando as análises das concentrações iniciais e residuais de dióxido de cloro e de peróxido de hidrogênio; ${ }^{12}$ determinação do rendimento em cada estágio de branqueamento, sendo que o rendimento da seqüência foi determinado multiplicando-se os rendimentos dos vários estágios.

O custo total de branqueamento foi calculado com base no custo total de reagentes, determinado a partir das dosagens de reagentes aplicadas em cada estágio das várias seqüências, em $\mathrm{kg}$ de reagente por $\mathrm{t}$ de polpa branqueada absolutamente seca ( $\mathrm{kg} / \mathrm{tas})$ e nos preços de cada reagente em US\$ (Tabela 2).

Tabela 2. Preços dos reagentes químicos de branqueamento

\begin{tabular}{lccccc}
\hline REAGENTE & $\mathrm{NaOH}$ & $\mathrm{ClO}_{2}$ & $\mathrm{H}_{2} \mathrm{SO}_{4}$ & $\mathrm{H}_{2} \mathrm{O}_{2}$ & $\mathrm{O}_{2}$ \\
\hline Preço, US\$/kg & 0,342 & 0,800 & 0,060 & 0,965 & 0,060 \\
\hline
\end{tabular}

\section{RESULTADOS E DISCUSSÃO}

As análises foram divididas em duas partes distintas, polpação kraft e branqueamento.

\section{Polpação kraft}

A influência da temperatura no teor de ácidos hexenurônicos na polpa com o mesmo fator $H$ (1127)

Os cozimentos foram realizados nas temperaturas de 170 e 156 ${ }^{\circ} \mathrm{C}$, com o mesmo fator $\mathrm{H}$ para atingir o número kappa de $17 \pm 1$. Os resultados são apresentados na Tabela 3.

Tabela 3. Cozimentos kraft realizados com álcali ativo $17 \%$, como $\mathrm{Na}_{2} \mathrm{O}$, em diferentes temperaturas

\begin{tabular}{lcc}
\hline Resultados & $156{ }^{\circ} \mathrm{C}$ & $170{ }^{\circ} \mathrm{C}$ \\
\hline Número kappa & 17,0 & 16,2 \\
Rendimento depurado, \% & 50,8 & 50,2 \\
Rendimento total, \% & 50,8 & 50,7 \\
Ácido hexenurônico, mmol/kg & 61,3 & 49,4 \\
pH & 12,6 & 12,8 \\
\hline
\end{tabular}

O rendimento depurado do cozimento kraft realizado a $156{ }^{\circ} \mathrm{C}$ foi superior em 0,6 ponto percentual ao cozimento realizado a 170 ${ }^{\circ} \mathrm{C}$, Tabela 3, em conformidade com os trabalhos dos autores ${ }^{13,14}$ mas, depende também do teor de álcali utilizado na polpação. ${ }^{15}$ Uma das principais explicações deste ganho de rendimento depurado foi resultado dos maiores teores dos ácidos hexenurônicos da polpa marrom proveniente do cozimento kraft realizado a $156^{\circ} \mathrm{C}$, em conformidade com os estudos. ${ }^{13,16}$ Ainda, os ácidos hexenurônicos impedem a despolimerização terminal das xilanas, ${ }^{16}$ aumentando o rendimento do cozimento kraft e, para aumentar a autenticidade dessa teoria, temos um $\mathrm{pH}$ final menor para o cozimento realizado a $156^{\circ} \mathrm{C}$, indicando um maior consumo de álcali. Este maior consumo é, provavelmente, devido à reação do álcali com os ácidos 4-O-metilglicurônicos formando os ácidos hexenurônicos durante o cozimento. ${ }^{2}$ Um outro estudo recente ${ }^{15}$ não encontrou uma relação linear entre os teores dos ácidos hexenurônicos com os teores de xilanas na polpa de celulose, concluindo que não é tão simples quanto dizer que um elevado teor 
de xilana resulta num elevado teor de ácido hexenurônico.

\section{Branqueamento}

Efeito dos ácidos hexenurônicos na branqueabilidade da polpa

Os resultados apresentados nas Tabelas 4 e 5 indicam que a remoção dos ácidos hexenurônicos com a implementação do estágio ácido apresentou um decréscimo na branqueabilidade (consumo de dióxido de cloro pelo número kappa da polpa deslignificada com oxigênio). Trabalho realizado com polpas kraft provenientes de diferentes processos de cozimentos apresentou, também, decréscimo na branqueabilidade das polpas, as quais tinham um menor teor de ácidos hexenurônicos. ${ }^{17}$

O estágio de dioxidação apresenta uma maior velocidade de reação com os ácidos hexenurônicos que o estágio de hidrólise ácida, ${ }^{18}$ embora o dióxido de cloro não reaja com os ácidos hexenurônicos, mas apenas o cloro e o ácido hipocloroso que são gerados no estágio de dioxidação. ${ }^{19}$ Deve-se considerar, também, que o estágio de hidrolise ácida remove uma pequena parte de lignina, a qual provavelmente é mais susceptível ao ataque dos reagentes químicos de branqueamento nos estágios posteriores. O mesmo raciocínio deve ser aplicado para as Tabelas 6 e 7.

Os resultados apresentados nas Tabelas 4 e 5 indicam que quanto menores os teores dos ácidos hexenurônicos presentes na polpa de celulose, após o estágio da deslignificação com oxigênio, menor é sua branqueabilidade.

O branqueamento das polpas provenientes dos cozimentos realizados em temperaturas diferentes $\left(170\right.$ e $\left.156{ }^{\circ} \mathrm{C}\right)$ apresentou diferentes resultados de branqueabilidade (Tabelas 4 a 7). Os teores de ácidos hexenurônicos foram mais elevados para a polpa oriunda do cozimento realizado em uma temperatura menor. Esta polpa apre-

Tabela 4. Seqüência de branqueamento $\mathrm{OD}(\mathrm{E}+\mathrm{P}) \mathrm{DD}$ utilizada como referência $170^{\circ} \mathrm{C}$

\begin{tabular}{lcccccc}
\hline \multirow{2}{*}{ Resultados } & \multicolumn{7}{c}{ Estágios de branqueamento } \\
& Inicial & $\mathrm{O}$ & $\mathrm{Do}$ & $\mathrm{E}+\mathrm{P}$ & $\mathrm{D}_{1}$ & $\mathrm{D}_{\mathrm{f}}$ \\
\hline $\mathrm{ClO}_{2}, \mathrm{~kg} / \mathrm{t}$ & - & - & 7,0 & - & 5,7 & 2,3 \\
Alvura A.D., \% ISO & 33,7 & 50,4 & 73,6 & 82,4 & 87,0 & 90,2 \\
Número Kappa & 16,2 & 9,2 & - & 4,3 & - & - \\
Ác. hexenurônicos, \\
mmol/kg & 49,4 & - & - & - & - & 2,4 \\
$\begin{array}{l}\text { Branqueabilidade, } \\
\mathrm{ClO}_{2} / \text { kappa (após } \mathrm{O}_{2} \text { ) }\end{array}$ & & & & & & \\
\hline
\end{tabular}

Tabela 5. Sequiência de branqueamento $\mathrm{OA}_{\mathrm{HT}} \mathrm{D}(\mathrm{E}+\mathrm{P}) \mathrm{DD}$ utilizada como referência $170{ }^{\circ} \mathrm{C}$

\begin{tabular}{|c|c|c|c|c|c|c|c|}
\hline \multirow{2}{*}{ Resultados } & \multicolumn{7}{|c|}{ Estágio de branquemento } \\
\hline & Inicial & $\mathrm{O}$ & $\mathrm{A}_{\mathrm{HT}}$ & $\mathrm{D}_{0}$ & $\mathrm{E}+\mathrm{P}$ & $\mathrm{D}_{1}$ & $D_{f}$ \\
\hline $\mathrm{ClO}_{2}, \mathrm{~kg} / \mathrm{t}$ & - & - & - & 5,2 & - & 5,7 & 2,0 \\
\hline $\begin{array}{l}\text { Alvura A.D., } \\
\% \text { ISO }\end{array}$ & 33,7 & 50,4 & 54,0 & 69,6 & 81,2 & 88,2 & 90,2 \\
\hline Número Kappa & 16,2 & 9,2 & 6,8 & - & 3,0 & - & - \\
\hline $\begin{array}{l}\text { Ác. hexenurônicos, } \\
\mathrm{mmol} / \mathrm{kg}\end{array}$ & 49,4 & - & 29,3 & - & - & - & 2,1 \\
\hline $\begin{array}{l}\text { Branqueabilidade, } \\
\mathrm{ClO}_{2} / \mathrm{kappa} \\
\left(\text { após } \mathrm{O}_{2}\right) \\
\end{array}$ & & & & 1,40 & & & \\
\hline
\end{tabular}

Tabela 6. Seqüência de branqueamento $\mathrm{OD}(\mathrm{E}+\mathrm{P}) \mathrm{DD}$ realizada na polpa teste $156^{\circ} \mathrm{C}$

\begin{tabular}{lcccccc}
\hline \multirow{2}{*}{ Resultados } & \multicolumn{6}{c}{ Estágios de branqueamento } \\
& Inicial & $\mathrm{O}$ & $\mathrm{Do}$ & $\mathrm{E}+\mathrm{P}$ & $\mathrm{D}_{1}$ & $\mathrm{D}_{\mathrm{f}}$ \\
\hline $\mathrm{ClO}_{2}, \mathrm{~kg} / \mathrm{t}$ & - & - & 7,7 & - & 5,7 & 1,5 \\
Alvura A.D., \% ISO & 31,5 & 50,3 & 73,4 & 82,7 & 88,1 & 90,0 \\
Número Kappa & 17,0 & 10,1 & - & 4,1 & - & - \\
$\begin{array}{l}\text { Ác. hexenurônicos, } \\
\text { mmol/kg }\end{array}$ & 61,3 & - & - & - & - & 2,4 \\
$\begin{array}{l}\text { Branqueabilidade, } \\
\mathrm{ClO}_{2} / \text { kappa (após } \mathrm{O}_{2} \text { ) }\end{array}$ & & & & & & \\
\hline
\end{tabular}

Tabela 7. Sequiência de branqueamento $\mathrm{OA}_{\mathrm{HT}} \mathrm{D}(\mathrm{E}+\mathrm{P}) \mathrm{DD}$ realizada na polpa teste $156^{\circ} \mathrm{C}$

\begin{tabular}{lccccccc}
\hline \multirow{2}{*}{ Resultados } & \multicolumn{7}{c}{ Estágio de branquemento } \\
& Inicial & $\mathrm{O}$ & $\mathrm{A}_{\mathrm{HT}}$ & $\mathrm{D}_{0}$ & $\mathrm{E}+\mathrm{P}$ & $\mathrm{D}_{1}$ & $\mathrm{D}_{\mathrm{f}}$ \\
\hline $\mathrm{ClO}_{2}, \mathrm{~kg} / \mathrm{t}$ & - & - & - & 4,9 & - & 5,7 & 0,8 \\
$\begin{array}{l}\text { Alvura A.D., } \\
\% \text { ISO }\end{array}$ & 31,5 & 50,3 & 54,0 & 73,1 & 82,2 & 88,9 & 90,3 \\
$\begin{array}{l}\text { Número Kappa } \\
\text { Ác. hexenurônicos, } \\
\text { mmol/kg }\end{array}$ & 17,0 & 10,1 & 6,5 & - & 2,6 & - & - \\
$\begin{array}{l}\text { Branqueabilidade, } \\
\text { ClO } / \text { kappa } \\
\text { (após } \mathrm{O}_{2} \text { ) }\end{array}$ & & - & 28,5 & - & - & - & 1,9 \\
& & & & & & & \\
\end{tabular}

sentou uma maior branqueabilidade para as sequiências $\mathrm{OD}(\mathrm{E}+\mathrm{P}) \mathrm{DD}$ e $\mathrm{OA}_{\mathrm{HT}} \mathrm{D}(\mathrm{E}+\mathrm{P}) \mathrm{DD}$, mostrando que um maior teor de ácido hexenurônico na polpa aumenta a sua branqueabilidade. Consequientemente, $\mathrm{o}$ número kappa devido aos ácidos hexenurônicos consome uma menor quantidade do dióxido de cloro que o mesmo número kappa devido à lignina residual da polpa. Mas, a velocidade da reação do dióxido de cloro com a lignina é duas vezes maior que com os ácidos hexenurônicos no estágio de dioxidação. ${ }^{18}$ Não se pode deixar de mencionar que uma maior temperatura de cozimento aumenta o teor de grupos $\mathrm{OH}$-alifáticos e carboxílicos, que favorecem a reatividade da lignina e, portanto, devem favorecer a branqueabilidade da polpa ${ }^{14}$ Embora neste mesmo trabalho a branqueabilidade da polpa oriunda de uma temperatura menor $\left(160^{\circ} \mathrm{C}\right)$ tenha sido maior que à $170^{\circ} \mathrm{C}$, utilizando a sequiência $\mathrm{D}(\mathrm{E}+\mathrm{O}) \mathrm{DD}$ para o branqueamento da polpa. ${ }^{14}$

Na Tabela 8 são apresentados os resultados das seqüências de branqueamento das amostras das polpas Kraft provenientes dos cozimentos realizados à 170 e $156^{\circ} \mathrm{C}$ pelas sequiências $\mathrm{OD}(\mathrm{E}+\mathrm{P})$ $\mathrm{DD}, \mathrm{OD}(\mathrm{E}+\mathrm{P}) \mathrm{DD}, \mathrm{OA}_{\mathrm{HT}} \mathrm{D}(\mathrm{E}+\mathrm{P}) \mathrm{DD} \mathrm{OA}_{\mathrm{HT}} \mathrm{D}(\mathrm{E}+) \mathrm{DP}$. O rendimento, consumo de reagentes, reversão de alvura, viscosidade e custo foram avaliados para o branqueamento da polpa de celulose na alvura de $90 \%$ ISO, Tabelas 8 e 9 .

Embora a perda de rendimento no estágio ácido seja de 1,8 ponto percentual, verifica-se que o rendimento total das sequiências de branqueamento diferencia em apenas 0,8 ponto percentual, Tabela 9 . Este fato deve ser devido à existência de carboidratos de baixo grau de polimerização na polpa, os quais já estão predispostos a serem solubilizados nos estágios de branqueamento. O principal efeito do tratamento ácido na celulose e nas hemiceluloses é reduzir o seu grau de polimerização, mas não reduz significativamente o rendimento do estágio de branqueamento. Os rendimentos dos diferentes processos de branqueamento ECF utilizados neste trabalho foram os mesmos 
Tabela 8. Consumo de reagentes do branqueamento pelo processo ECF

\begin{tabular}{|c|c|c|c|c|c|}
\hline Seqüências & $\begin{array}{l}\mathrm{O}_{2}, \\
\mathrm{~kg} / \mathrm{t}\end{array}$ & $\begin{array}{c}\mathrm{ClO}_{2}, \\
\mathrm{~kg} / \mathrm{t}\end{array}$ & $\begin{array}{c}\mathrm{H}_{2} \mathrm{SO}_{4}, \\
\mathrm{~kg} / \mathrm{t}\end{array}$ & $\begin{array}{c}\mathrm{NaOH}, \\
\mathrm{kg} / \mathrm{t}\end{array}$ & $\begin{array}{c}\mathrm{H}_{2} \mathrm{O}_{2}, \\
\mathrm{~kg} / \mathrm{t}\end{array}$ \\
\hline $\mathrm{OD}(\mathrm{E}+\mathrm{P}) \mathrm{DD}\left(170^{\circ} \mathrm{C}\right)$ & 20,0 & 15,0 & 6,0 & 33,4 & 3,0 \\
\hline $\mathrm{OD}(\mathrm{E}+\mathrm{P}) \mathrm{DP}\left(170^{\circ} \mathrm{C}\right)$ & 20,0 & 12,7 & 6,0 & 39,0 & 6,0 \\
\hline $\mathrm{OA}_{\mathrm{HT}} \mathrm{D}(\mathrm{E}+\mathrm{P}) \mathrm{DD}\left(170^{\circ} \mathrm{C}\right)$ & 20,0 & 12,9 & 11,0 & 35,0 & 3,0 \\
\hline $\mathrm{OA}_{\mathrm{HT}} \mathrm{D}(\mathrm{E}+\mathrm{P}) \mathrm{DP}\left(170^{\circ} \mathrm{C}\right)$ & 20,0 & 11,3 & 11,0 & 41,0 & 5,0 \\
\hline $\mathrm{OD}(\mathrm{E}+\mathrm{P}) \mathrm{DD}\left(156^{\circ} \mathrm{C}\right)$ & 20,0 & 14,9 & 6,0 & 33,0 & 3,0 \\
\hline $\mathrm{OD}(\mathrm{E}+\mathrm{P}) \mathrm{DP}\left(156^{\circ} \mathrm{C}\right)$ & 20,0 & 13,4 & 6,0 & 39,0 & 6,0 \\
\hline $\mathrm{OA}_{\mathrm{HT}} \mathrm{D}(\mathrm{E}+\mathrm{P}) \mathrm{DD}\left(156^{\circ} \mathrm{C}\right)$ & 20,0 & 11,4 & 11,0 & 34,3 & 3,0 \\
\hline $\mathrm{OA}_{\mathrm{HT}} \mathrm{D}(\mathrm{E}+\mathrm{P}) \mathrm{DP}\left(156^{\circ} \mathrm{C}\right)$ & 20,0 & 10,6 & 11,0 & 41,0 & 4,5 \\
\hline
\end{tabular}

para as polpas provenientes de diferentes temperaturas de cozimento. Isto indica que o maior rendimento obtido no cozimento realizado numa menor temperatura permanece durante o processo de branqueamento, conseqüentemente, sendo mais atrativo financeiramente.

Os reagentes químicos, hidróxido de sódio, ácido sulfúrico e dióxido de cloro, utilizados nas quatro seqüências de branqueamento para atingir uma alvura de 90,0\% ISO, apresentaram diferentes consumos, Tabela 8.

O consumo de hidróxido de sódio foi sensivelmente maior para a seqüência de branqueamento com o estágio ácido, devido à necessidade da neutralização após o estágio de hidrólise ácida.

Os consumos de ácido sulfúrico foram de $6 \mathrm{~kg} / \mathrm{t}$ para as seqüências $\mathrm{OD}(\mathrm{E}+\mathrm{P}) \mathrm{DD}, \mathrm{OD}(\mathrm{E}+\mathrm{P}) \mathrm{DP}$ e de $11 \mathrm{~kg} / \mathrm{t}$ para as $\mathrm{OA}_{\mathrm{HT}} \mathrm{D}(\mathrm{E}+\mathrm{P}) \mathrm{DD}$, $\mathrm{OA}_{\mathrm{HT}} \mathrm{D}(\mathrm{E}+\mathrm{P}) \mathrm{DP}$, independentemente da temperatura utilizada no cozimento da polpa. A implementação do estágio de hidrólise ácida demanda um maior consumo de ácido, mas este reagente apresenta baixo custo em relação ao dióxido de cloro.

Os consumos de dióxido de cloro utilizado pelas sequiências de branqueamento foram valores tradicionais para os processos de branqueamentos ECF, Tabela 8. Com a implementação do estágio de hidrólise ácida, $\mathrm{OA}_{\mathrm{HT}} \mathrm{D}(\mathrm{E}+\mathrm{P}) \mathrm{DD}$, obteve-se uma economia de 14 e $23 \%$ no consumo de dióxido de cloro, respectivamente, para as polpas provenientes dos cozimentos nas temperaturas de 170 e $156^{\circ} \mathrm{C}$. Os ácidos hexenurônicos presentes na polpa consomem dióxido de cloro, mas com a implementação do estágio ácido, proporcionam a remoção dos ácidos hexenurônicos da polpa, reduzindo conseqüentemente o consumo de dióxido de cloro necessário ao branqueamento da polpa a 90,0\% ISO.

A seqüência $\mathrm{OA}_{\mathrm{HT}} \mathrm{D}(\mathrm{E}+\mathrm{P}) \mathrm{DD}$ apresentou uma reversão de alvura de $2,0 \%$ ISO, enquanto que na sequiência OD(E+P)DD foi de $2,2 \%$ ISO, Tabela 9. Esta diferença na reversão de alvura é devida principalmente ao fato da introdução do estágio ácido, a quente, favorecendo a eliminação dos ácidos hexenurônicos da polpa,${ }^{20}$ que citam uma forte ligação da quantidade de ácidos hexenurônicos com a reversão de alvura da polpa. As seqüências de branqueamento com o último estágio, o peróxido de hidrogênio em substituição ao estágio de dióxido de cloro, apresentaram uma menor reversão de alvura. Isto se deve a maior estabilidade dos grupos presentes na celulose quando são branqueados com peróxido de hidrogênio em meio alcalino. ${ }^{21}$

Embora a implementação do estágio ácido tenha causado um decréscimo na viscosidade de $13,6 \%$, a diferença entre a viscosidade final das duas sequiências, $\mathrm{OD}(\mathrm{E}+\mathrm{P}) \mathrm{DD}$ e $\mathrm{OA}_{\mathrm{HT}} \mathrm{D}(\mathrm{E}+\mathrm{P}) \mathrm{DD}$, foi de apenas 5,3 pontos percentuais. Deve-se notar, ainda, que a viscosidade final da seqüência $\mathrm{OA}_{\mathrm{HT}} \mathrm{D}(\mathrm{E}+\mathrm{P}) \mathrm{DD}$ foi de $19,7 \mathrm{mPa} . \mathrm{s}$, a qual se apresenta dentro dos padrões de qualidade exigida. É importante esclarecer que o principal efeito negativo do estágio ácido na polpa é o decréscimo da viscosidade. ${ }^{22}$ Nota-se que o estágio de peróxido de hidrogênio é mais deletério para a viscosidade que o estágio de dióxido de cloro, como já está bem estabelecido conforme os autores Oliveira e Colodette. ${ }^{20}$

A sequiência $\mathrm{OD}(\mathrm{E}+\mathrm{P}) \mathrm{DD}$ foi utilizada como referência, atribuindo o custo relativo de $100 \%$. A implementação do estágio de peroxidação elevou sensivelmente o custo do branqueamento de 100 para $111 \%$, enquanto que o estágio ácido apresentou um sensível decréscimo no custo (Tabela 9). ${ }^{23}$ As polpas produzidas em diferentes temperaturas de cozimento apresentaram custo de branqueamento semelhante para a sequiência $\mathrm{OD}(\mathrm{E}+\mathrm{P}) \mathrm{DD}$. A implementação do estágio ácido no processo de branqueamento da polpa proveniente do cozimento realizado em uma temperatura menor resultou em um menor custo químico para o branqueamento, devido ao menor consumo de dióxido de cloro.

\section{CONCLUSÃO}

A partir dos resultados obtidos neste trabalho, concluiu-se que uma temperatura menor no cozimento aumenta o rendimento e a branqueabilidade da polpa kraft, com número kappa semelhante. Um maior teor de ácidos hexenurônicos na polpa após a deslignificação com oxigênio aumenta a branqueabilidade da polpa. O estágio ácido promoveu uma economia de $23 \%$ no consumo do dióxido de cloro utilizado na seqüência $\mathrm{OA}_{\mathrm{HT}} \mathrm{D}(\mathrm{E}+\mathrm{P}) \mathrm{DD}$, mostrando ser um importante meio de redução de custo ou aumento da produção da polpa branqueada. Apresentou efeito positivo na reversão de alvura da polpa branqueada, mas com um pequeno efeito negativo no rendimento e viscosidade da polpa. A substituição do estágio realizado com dióxido de cloro pelo peróxido de hidrogênio resultou em uma polpa com uma menor reversão de alvura, mas com um maior custo relativo.

\section{AGRADECIMENTOS}

À FAPESP - Fundação de Amparo à Pesquisa do Estado de São Paulo, processo número: 2007/53872-4 - pelo apoio financeiro.

Tabela 9. Resultados do branqueamento pelo processo ECF

\begin{tabular}{|c|c|c|c|c|c|}
\hline Seqüências & Rend., $\%$ & Viscos.,mPa.s & Hexenurônicos, $\mathrm{mmol} / \mathrm{kg}$ & Reversão alvura, \% ISO & Custo relativo $\%$ \\
\hline $\mathrm{OD}(\mathrm{E}+\mathrm{P}) \mathrm{DD}\left(170^{\circ} \mathrm{C}\right)$ & 95,2 & 17,4 & 2,4 & 2,9 & 100 \\
\hline $\mathrm{OD}(\mathrm{E}+\mathrm{P}) \mathrm{DP}\left(170^{\circ} \mathrm{C}\right)$ & 95,0 & 17,1 & 3,0 & 2,0 & 111 \\
\hline $\mathrm{OA}_{\mathrm{HT}} \mathrm{D}(\mathrm{E}+\mathrm{P}) \mathrm{DD}\left(170^{\circ} \mathrm{C}\right)$ & 94,4 & 17,0 & 2,1 & 2,5 & 97 \\
\hline $\mathrm{OA}_{\mathrm{HT}} \mathrm{D}(\mathrm{E}+\mathrm{P}) \mathrm{DP}\left(170^{\circ} \mathrm{C}\right)$ & 94,3 & 16,8 & 2,3 & 1,9 & 107 \\
\hline $\mathrm{OD}(\mathrm{E}+\mathrm{P}) \mathrm{DD}\left(156^{\circ} \mathrm{C}\right)$ & 95,2 & 20,8 & 2,4 & 2,2 & 99 \\
\hline $\mathrm{OD}(\mathrm{E}+\mathrm{P}) \mathrm{DP}\left(156^{\circ} \mathrm{C}\right)$ & 95,0 & 20,1 & 2,8 & 1,8 & 113 \\
\hline $\mathrm{OA}_{\mathrm{HT}} \mathrm{D}(\mathrm{E}+\mathrm{P}) \mathrm{DD}\left(156^{\circ} \mathrm{C}\right)$ & 94,4 & 19,7 & 1,9 & 2,0 & 92 \\
\hline $\mathrm{OA}_{\mathrm{HT}} \mathrm{D}(\mathrm{E}+\mathrm{P}) \mathrm{DP}\left(156^{\circ} \mathrm{C}\right)$ & 94,2 & 19,3 & 2,1 & 1,4 & 103 \\
\hline
\end{tabular}




\section{REFERENCIAS}

1. Browning, B. L.; Methods of wood chemistry, Wiley-Interscience: New York, 1967.

2. Chakar, F.; Allison, L.; Ragauskas, T.; Mcdonough, J.; Sezgi, U.; Tappi J. 2000, 83, 62 .

3. Jiang, Z. H.; Lierop, B. V.; Berry, R.; Tappi J. 2000, 83, 167.

4. Vuorinen, T.; Burchert, J.; Teleman, A.; Tenkanen, M.; Fagerstrom, P.; Proceedings of the 1996 International Pulp Bleaching Conference, Washington D.C., USA, 1996.

5. Gustavsson, C. A-S.; AL-dajani, W. W.; Nord. Pulp Pap. Res. J. 2000 , 15,160 .

6. Pettersson, E. A. K.; Ragnar, M.; Lindstöm, M. E.; Nord. Pulp Pap. Res. J. 2002, 17, 222

7. Devenyns, J.; Chauveheid, E.; Proceedings of the $9^{\text {th }}$ International Symposium on Wood and Pulping Chemistry, Montreal, Canada, 1997.

8. Dahlman, O.; Morck, R.; Larsson, P. T.; Proceedings of the 1996 International Pulp Bleaching Conference, Washington D.C., USA, 1996.

9. Teleman, A.; Hausalo, T.; Tenkanen, M.; Vuorinen, T.; Carbohydr. Res. 1996, 2, 197.

10. TAPPI - Technical Association of the Pulp and Paper Industry; Test methods; Atlanta, USA. 2000-2001. TAPPI PRESS, CD-ROM, 2000.

11. Canadian Pulp and Paper Association; Technical section standard test methods, Montreal: 1986.
12. Kraft, F.; Pulps and paper manufacture, $3^{\text {rd }}$ ed., McGraw-Hill: New York, 1967.

13. Monrroy, M.; Valdebenito, M.; Baeza, J.; Mendonça, R.; Freer, J.; Anais do $39^{\circ}$ Congresso e Exposição Internacional de Celulose e Papel, São Paulo, Brasil, 2006.

14. Gomide, J. L.; Colodette, J. L.; Oliveira, R. C.; Girard, R.; Argyropoulos, D. S.; O Papel 2000, 12, 61.

15. Johansson, D.; Germgard, U.; O Papel 2006, 10, 84.

16. Chai, X. S.; Luo, Q.; Yoon, S. H.; Zhu, J. Y.; Proceedings of the $11^{\text {th }}$ International Symposium on Wood and Pulping Chemistry, Nice, France, 2001.

17. Daniel, A. I. D.; Neto, C. P.; Evtuguin, D. V.; Silvestre, A. J. D.; Tappi J. 2003, 2, 3 .

18. Ventorim, G.; Tese de Doutorado, Universidade Federal de Viçosa, Brasil, 2004.

19. Costa, M. M.; Tese de Doutorado, Universidade Federal de Viçosa, Brasil, 2001.

20. Eiras, K. M. M.; Colodette, J. L.; J. Pulp Pap. Sci. 2003, 29, 64.

21. Oliveira, R. L.; Colodette, J. L.; Eiras, K. M. M.; Ventorim, G.; Rev. Arvore 2006, 30, 439.

22. Shackford, L.; Santos, C. A.; Colodette, J. L.; Anais do $33^{\circ}$ Congresso Internacional de Celulose e Papel, São Paulo, Brasil, 2000.

23. Lindström, L. A.; Larsson, P. E.; Proceedings of the International Colloquium on Eucalyptus Kraft Pulp, Viçosa, Brasil, 2003. 